\title{
FURTHER OBSERVATIONS ON A PROBLEM OF CONSTANT SURFACE HEATING OF A VARIABLE-CONDUCTIVITY HALFSPACE*
}

\author{
$\mathrm{BY}$ \\ LEONARD Y. COOPER \\ Bell Telephome Laboratories, Inc. Whippany, New Jersey
}

\begin{abstract}
A solution to the problem of constant surface heating of an initially constant-temperature, $T_{0}^{*}$, halfspace where the material in question has a temperaturedependent thermal conductivity is obtained. The thermal conductivity, $k^{*}$, is specifically given by $k^{*}=k_{0}^{*} \exp \left[\lambda\left(T^{*}-T_{0}^{*}\right) / T_{0}^{*}\right]$. The solution is valid for both heating and cooling of the material where $\lambda$ and $k_{0}^{*}$ are arbitrary in magnitude, and $\lambda$ can be either positive or negative in sign.

As in a previous investigation of this problem, the present work studies the application of integration techniques of the boundary layer equations of fluid mechanics to the solution of a nonlinear diffusion problem of heat conduction. Exact results obtained here for the first three variable coefficients of an expansion of $\phi=k^{*} / k_{0}^{*}$ about its initial state give increased confidence in the success of the previously utilized approximate solution technique, and, in fact, suggest an improved approximate technique for treating the type of problem under consideration. This latter technique is developed herein. It is finally concluded that the exact solutions for the first three terms of the $\phi$ expansion mentioned above can be used to construct a closed-form approximate solution to the problem which is useful over a significant range of the independent variables.
\end{abstract}

1. Introduction. This work will treat the solution to the problem of constant surface heating, $H^{*}$, of an initially constant-temperature halfspace. We will consider that the thermal conductivity, $k^{*}$, of the material in question can be accurately represented by ${ }^{1}$

$$
\phi=k^{*} / k_{0}^{*}=\exp \left[\lambda\left(T^{*}-T_{0}^{*}\right) / T_{0}^{*}\right]=\exp V
$$

where $\lambda$ is arbitrary and $k_{0}^{*}$ is the thermal conductivity at the initial uniform temperature $T_{\mathbf{0}}^{*}$, of the halfspace. Further, the material property $\rho^{*} C^{*}$, where $\rho^{*}$ is the density and $C^{*}$ the specific heat, will be assumed to be accurately represented as a constant.

The boundary value problem describing the above phenomenon in terms of the dimensionless conductivity, $\phi$, is given [1] as

$$
\begin{gathered}
\phi \phi_{\eta \eta}+\eta \phi_{\eta}=\zeta \phi_{\zeta}, \\
\lim _{\eta \rightarrow 0 ; \zeta \text { fixed }} \phi_{\eta}=\zeta, \\
\lim _{\eta \rightarrow \infty ; \zeta \text { fixed }} \phi=1 .
\end{gathered}
$$

* Received December 17, 1969; revised version received July 1, 1970. This work was supported by the U. S. Army under Contract DA-30-069-AMC-333(Y).

${ }^{1}$ Starred and unstarred quantities always refer to dimensional and dimensionless quantities respectively. 
where the dimensionless independent variables $\eta$ and $\zeta$ are defined as

$$
\eta=x^{*}\left[\rho^{*} C^{*} /\left(2 k_{0}^{*} t^{*}\right)\right]^{1 / 2}, \quad \zeta=-\lambda H^{*}\left[2 t^{*} /\left(\rho k_{0}^{*} C^{*}\right)\right]^{1 / 2} / T_{0}^{*},
$$

and where $x^{*}$ and $t^{*}$ are depth into the halfspace and time after initiation of the heating respectively. Once $\phi$ has been obtained from the above, the dimensionless temperature, $V$, can readily be obtained from Eq. (1).

A solution to the problem of Eqs. (2)-(4) has been obtained by Cooper [1]. In that work the solution was obtained by extending a solution technique which was developed by Meksyn [2 pp. 98ff] for integration of the boundary layer equations of fluid mechanics. Thus, in reference [1] the solution proceeded by relating the solution form $\phi=\sum a_{n}(\zeta) \eta^{n}$, which satisfied the condition of (3), with the form $\left[\sum \psi_{n}(\zeta) \eta^{n}\right] \exp \left[-\eta^{2} / 2\right]$ for $\phi_{n}$. The present work will treat the identical problem from the point of view of expanding $\phi$ as $\phi=\sum b_{n}(\eta) \zeta^{n}$. Thus, the solution to the nonlinear diffusion problem under consideration will be treated here in a manner reminiscent, say, of the Görtler [3], [4] solution to the boundary layer problem of fluid mechanics. As will be seen, the latter expansion of $\phi$ will allow the $b_{n}$ 's to be generally represented by solutions to nonhomogeneous, linear, ordinary differential equations with appropriate boundary conditions. These indicate that all the $b_{n}$ 's are, in fact, entire analytic functions. Although the general function cannot be practically obtained in any other than the form of quadratures presented herein, the first few $b_{n}$ 's are explicitly found. These shed light on the success of the original solution to this problem as given in [1]. Moreover, the original solution technique used there is recast here in terms of obtaining approximate solutions for the $b_{n}$ 's under consideration directly from the governing differential equations and boundary conditions mentioned above. Finally, as will be seen, the quadrature solution form for the $b_{n}$ obtained here also suggests a second and more direct technique, again through use of the ideas brought forth by Meksyn, for an approximate evaluation of the unknown functions $b_{n}$. Thus, although the exact solution to more than a few of the $b_{n}$ 's may not be practical, approximate solutions for these functions can be obtained through use of a Meksyn integration technique.

2. The solution. In view of the above introduction we seek a solution to the problem of Eqs. (2)-(4) by assuming the following expansion for the function $\phi$ :

$$
\phi=\sum_{n=0}^{\infty} b_{n}(\eta) \zeta^{n}
$$

Using this in Eqs. (2)-(4) we obtain separate boundary value problems for the $b_{n}$. The problem for $b_{0}$, specifically, becomes

$$
b_{0}(\eta) b_{0}^{\prime \prime}(\eta)+\eta b_{0}^{\prime}(\eta)=0 ; \quad b_{0}^{\prime}(0)=0 ; \quad b_{0}(\infty)=1 .
$$

Although the above equation is nonlinear the boundary conditions allow for the simple solution

$$
b_{0}(\eta)=1
$$

Using this result for $b_{0}$, the problems governing the solutions for subsequent $b_{n}$ can be given as

$$
b_{n}^{\prime \prime}(\eta)+\eta b_{n}^{\prime}(\eta)-n b_{n}(\eta)=-\sum_{m=1}^{n-1} b_{m}(\eta) b_{n-m}^{\prime \prime}(\eta), \quad n>0,
$$




$$
\begin{gathered}
b_{1}^{\prime}(0)=1, \quad \text { all other } b_{n}^{\prime}(0)=0, \\
b_{n}(\infty)=0 .
\end{gathered}
$$

It is clear from the above that each $b_{n}$ is governed in general by a linear nonhomogeneous ordinary differential equation where the nonhomogeneous parts are functions of the $b_{m}$ 's, $m<n$. Thus, we would expect that all the $b_{n}$ 's can, in principle, be exactly obtained recursively.

In order to cast the differential equations of (8) in a more usable form Kamke [5] suggests the following transformation to new dependent variables, $g_{n}(\eta)$ :

$$
b_{n}(\eta)=g_{n}(\eta) \exp \left[-\eta^{2} / 4\right], \quad n>0 .
$$

Using this in Eqs. (8)-(10), we obtain the boundary value problems which govern the $g_{n}$ :

$$
\begin{gathered}
g_{n}^{\prime \prime}(\eta)+\left[-(n+1)+\frac{1}{2}-\eta^{2} / 4\right] g_{n}(\eta)=h_{n}(\eta), \quad n>0, \\
g_{1}^{\prime}(0)=1, \text { all other } g_{n}^{\prime}(0)=0, \\
\lim _{\eta \rightarrow \infty} g_{n}(\eta) \exp \left[-\eta^{2} / 4\right]=0,
\end{gathered}
$$

where the functions $h_{n}$ are given as

$$
\begin{aligned}
h_{1}(\eta) & =0, \\
h_{n}(\eta) & =-\exp \left[-\eta^{2} / 4\right] \sum_{m=1}^{n-1} g_{m}(\eta)\left[g_{n-m}^{\prime \prime}(\eta)-\eta g_{n-m}^{\prime}(\eta)+\left(\eta^{2}-2\right) g_{n-m}(\eta) / 4\right] \\
& =\exp \left[\eta^{2} / 4\right] \sum_{m=1}^{n-1} b_{m}(\eta) b_{n-m}^{\prime \prime}(\eta), \quad n>1 .
\end{aligned}
$$

The solution to Eqs. (12)-(14) for $n=1$ is given by

$$
g_{1}(\eta)=-(2 / \pi)^{1 / 2} D_{-2}(\eta)
$$

where $D_{n}(\eta)$ is the $n$th order Weber-Hermite function [6]. For $n>1$ a quadrature form of solution to Eqs. (12)-(14) has been found to be

$$
\begin{aligned}
g_{n}(\eta)=(-1)^{(n+2) / 2}\left[D_{n}(i \eta) \int_{\eta}^{\infty}\right. & D_{-n-1}(\mu) h_{n}(\mu) d \mu \\
& \left.+D_{-n-1}(\eta) \int_{0}^{\eta} D_{n}(i \mu) h_{n}(\mu) d \mu\right] \text { for } n>1 \text { and even, } \\
& \begin{aligned}
g_{n}(\eta)=i(-1)^{(n-1) / 2} D_{n}(i \eta) \int_{\eta}^{\infty} & D_{-n-1}(\mu) h_{n}(\mu) d \mu \\
& +D_{-n-1}(\eta)\left\{i(-1)^{(n-1) / 2} \int_{0}^{\eta} D_{n}(i \mu) h_{n}(\mu) d \mu\right. \\
& \left.-(2 / \pi)^{1 / 2} n ! \int_{0}^{\infty} D_{-n-1}(\mu) h_{n}(\mu) d \mu\right\} \text { for } n>1 \text { and odd. }
\end{aligned}
\end{aligned}
$$

Other than obtaining the explicit solution for $g_{1}$ we have also found solutions for $g_{2}$ and $g_{3}$ from the above. These are 


$$
\begin{aligned}
g_{2}(\eta)= & (3 / 4)(2 / \pi)^{1 / 2}\left[1-(2 / 3)(2 / \pi)^{1 / 2} D_{0}(\eta) D_{-1}(\eta)\right] D_{-3}(\eta), \\
g_{3}(\eta)= & -(1 / 48)(2 / \pi)^{1 / 2}\left\{28-(2 / \pi)\left[27(3)^{1 / 2}-32\right]\right\} D_{-4}(\eta) \\
& +3^{1 / 2}(3 / 32)(2 / \pi)^{3 / 2}\left(\eta^{3}+3 \eta\right) D_{0}\left(2^{1 / 2} \eta\right) D_{-1}\left(3^{1 / 2} \eta\right) \\
& -(1 / 288)(2 / \pi)^{3 / 2} D_{0}^{2}(\eta)\left[4\left(14+9 \eta^{2}\right) D_{0}^{3}(\eta)\right. \\
& -18\left(\eta+\eta^{3}\right) D_{0}^{2}(\eta) D_{-1}(\eta)+3\left(5+22 \eta^{2}+3 \eta^{4}\right) D_{0}(\eta) D_{-1}^{2}(\eta) \\
& \left.-8\left(3 \eta+4 \eta^{3}\right) D_{-1}^{3}(\eta)\right]-(1 / 16)(2 / \pi) D_{0}(\eta)\left[3 \eta D_{0}^{2}(\eta)\right. \\
& \left.-\left(5+7 \eta^{2}\right) D_{0}(\eta) D_{-1}(\eta)+2\left(3 \eta+2 \eta^{3}\right) D_{-1}^{2}(\eta)\right] .
\end{aligned}
$$

The task of obtaining explicit solutions for each of the $g_{n}$ 's from Eqs. (17) and (18) is clearly one of evaluating two indefinite integrals $\int D_{n}(i \mu) h_{n}(\mu) d \mu$ and $\int D_{-n-1}(\mu) h_{n}(\mu) d \mu$. As it happens, with increasing $n$ the effort required in the evaluation of these quickly increases. Moreover, it is clear that the exact solution for these integrals for each $n$ is a necessary prerequisite for obtaining both the particular $g_{n}$ under consideration and all subsequent $g_{n}$ 's. Although we have obtained the $g_{n}$ 's (and, as a consequence of Eq. (11), the $b_{n}$ 's) only for $n=0,1,2$, and 3 , the existence of all the $g_{n}$ 's (and, therefore, the $b_{n}$ 's) as entire analytic functions can nevertheless be shown. The definite knowledge of such existence will, of course, add credence to our subsequent attempts at obtaining approximate solutions for these functions.

That the $g_{n}$ 's are, in fact, entire functions can be shown from the solution form of Eqs. (17) and (18). A discussion to this end proceeds as follows:

Consider the situation for $g_{n}, n=p>1$. Then assume that all the $g_{m}$ 's, $m<p$ have been obtained and are found to be entire functions. From Eq. (15) it is then clear that $h_{p}$ is an entire function. Furthermore, $D_{p}(i \eta)$ and $D_{-p-1}(\eta)$ are also entire functions of $\eta$. It follows from Eqs. (17) and (18) that $g_{p}$ is an entire function provided the existence of $\int_{n}^{\infty} D_{-p-1}(\mu) h_{p}(\mu) d \mu$ can be demonstrated. From the boundary condition of Eq. (10), satisfied by the $b_{m}, m<p$, it follows from Eq. (11) that for $\eta \rightarrow \infty h_{p}(\eta)=o\left(\exp \left[\eta^{2} / 4\right]\right)$. Also [6], $D_{-p-1}(\eta)=O\left(\eta^{-p-1} \exp \left[-\eta^{2} / 4\right]\right)$ as $\eta \rightarrow \infty$. Thus, for $\eta \rightarrow \infty, D_{-p-1}(\eta) h_{p}(\eta)=$ $O\left(\eta^{-D-1} \exp \left[-\eta^{2} / 4\right]\right), o\left(\exp \left[-\eta^{2} / 4\right]\right)=o\left(\eta^{-D-1}\right)$. In view of all the above this asymptotic character of $D_{-p-1}(\eta) h_{p}(\eta)$ then demonstrates the existence of the aforementioned integral. Moreover, $g_{1}$ has been found to be an entire function. It follows from induction that all the $g_{n}$ 's are entire functions.

3. Approximate solutions for the $b_{n}(\eta)$ from the governing equations. We now sketch an approximate solution technique for obtaining the $b_{n}$ 's which follows, and is essentially equivalent to, the Meksyn solution technique utilized in [1].

Considering expansions of the $b_{n}$ about $\eta=0$, we define

$$
b_{n}(\eta)=\sum_{m=0}^{\infty} A_{m, n} \eta^{m} .
$$

The result of Eq. (7) and the conditions of Eqs. (9) clearly indicate that

$$
\begin{aligned}
& A_{0,0}=1, \quad \text { all other } A_{m, 0}=0, \\
& A_{1,1}=1, \quad \text { all other } A_{1, m}=0 .
\end{aligned}
$$

Let us assume that all $b_{q}(0)=A_{0, a}, q<n$ have been obtained. (Note that all $b_{q}(0)=A_{0 . a}$ of this work are identical to the $A_{0, a}=\alpha_{a}$ of reference [1].) Then, from recurrence re- 
lationships readily obtainable from Eq. (8) with the use of Eqs. (21) and (22), this therefore implies the knowledge of all $A_{p, a}, q<n$ and, in fact, the $b_{a}, q<n$ themselves. These recurrence relationships are explicitly given in Eq. (17) of [1].

For a given $n$ we now seek the value of $b_{n}(0)=A_{0, n}$. Once this has been obtained then all of the other unknown $A_{p, n}$ can be obtained from the recurrence relations mentioned above. A solution for $b_{n}(0)$ therefore implies a solution to the unknown function $b_{n}(\eta)$ itself. According to the boundary condition of (10), it is reasonable to assume that as $\eta \rightarrow \infty$ the term $n b_{n}(\eta)-\sum_{m-1}^{n} b_{m}(\eta) b_{m-n}^{\prime \prime}(\eta) \equiv f_{n}(\eta)$ goes to zero. Let us assume that as $\eta \rightarrow \infty, f_{n}(\eta)=o\left[\eta b_{n}^{\prime}(\eta)\right]$. Then asymptotically, for large $\eta$, Eqs. (8)-(10) yield $b_{n}^{\prime}(\eta) \sim \Psi_{n}(\eta) \exp \left[-\eta^{2} / 2\right]$ where $\psi_{n}$ is essentially constant at large $\eta$. This suggests the following form for $b_{n}^{\prime}(\eta)$ :

$$
b_{n}^{\prime}(\eta)=\psi_{n}(\eta) \exp \left[-\eta^{2} / 2\right] .
$$

We expand the $\psi_{n}$ about $\eta=0$ and for later convenience take the expanded form for the entire function $b_{n}^{\prime}(\eta)$ as

$$
b_{n}^{\prime}(\eta)=\exp \left[-\eta^{2} / 2\right] \sum_{m=0}^{\infty}\left\{2^{(1-m) / 2} G_{m, n} \eta^{m} / \Gamma[(m+1) / 2]\right\}
$$

where $\Gamma(x)$ is the gamma function. By placing the assumed expansion form for $b_{n}$ as per Eq. (21) into the left-hand side of Eq. (23), multiplying both sides of this latter equation by $\exp \left[\eta^{2} / 2\right]$, and expanding this exponential function about $\eta=0$, it is possible in the end to obtain an equation for the $G_{n, m}$ as a function of the (known) $A_{p, q}$ and the unknown constant $b_{n}(0)$ of interest. We are specifically able to find that $G_{m, n}=\mathcal{C}_{m, n} b_{n}(0)+\mathfrak{F}_{m, n}$ where the $\mathcal{C}_{m, n}$ are constants and the $\mathfrak{F}_{m, n}$ are functions of the $A_{p, a}, p<n, q<m$. These are given in Eq. (18) of [1].

We are now in a position to integrate Eq. (23) formally. Using the condition of Eq. (10) we find

$$
b_{n}(0) \sim-\int_{0}^{\infty} \exp \left[-\eta^{2} / 2\right] \sum_{m=0}^{\infty}\left\{2^{(1-m) / 2} G_{m, n} \eta^{m} / \Gamma[(m+1) / 2]\right\} d \eta .
$$

We use the $\sim$ from here on to indicate that the right-hand side is only "a representation" of the left-hand side since the integrand on the right is itself not defined at the upper limit of integration. We formally exchange integration and summation in this last and eventually obtain

$$
b_{n}(0) \sim-\sum_{m=0}^{\infty} G_{m, n}=-\sum_{m=0}^{\infty}\left[\mathfrak{C}_{m, n} b_{n}(0)+\mathfrak{F}_{m, n}\right] .
$$

The problem has now been reduced to one of obtaining a meaningful equation for the $b_{n}(0)$ from Eq. (25). The identical problem comes about in [1], and the situation is discussed at length in that work. There it was found that although the infinite sums of Eq. (25) are in general divergent it is consistent to assume that they can be rendered convergent by the use of the multiple Euler transformation [2] [7] $\varepsilon^{(p)}$ defined by

$$
\begin{aligned}
\mathcal{E}\left(\mathfrak{N}_{n, m}\right) & \equiv \mathcal{E}^{(1)}\left(\mathfrak{N}_{n, m}\right) \equiv 2^{-(n+1)} \sum_{a=0}^{n} \Re_{a, m}\{n ! /[(n-q) ! q !]\}, \\
\mathcal{E}^{(\mathcal{D})}\left(\mathfrak{N}_{n, m}\right) & \equiv \mathcal{E}\left[\mathcal{E}^{(\mathcal{D}-1)}\left(\mathfrak{T}_{n, m}\right)\right], \quad p>1 .
\end{aligned}
$$


Thus the validity of an assumption of divergence, asymptotic divergence, or convergence of the infinite sum $\sum_{m=0}^{\infty} G_{m, n}$ or any one of its multiply transformed infinite sums, $\sum_{m=0}^{\infty} \mathcal{E}^{(\mathcal{D})}\left[G_{m, n}\right]$, could only be studied $a$ posteriori after a tentative solution for the $b_{n}(0)$ under consideration was obtained. This was true, of course, since the terms $G_{m, n}$ contain the unknown, $b_{n}(0)$, itself.

A summary of all the results for the $b_{n}(0)$ which were obtained in [1] are reproduced here in Table 1.

In the next section we will seek solutions for the $b_{n}(0)=g_{n}(0)$ directly from our formal $g_{n}(\eta)$ solution form given in Eqs. (17) and (18). As will be seen, this will enable us to represent a given $g_{n}(0)$ in terms of an infinite sum of known terms, the divergence or convergence of which can be studied previous to any actual summing process. In the event that divergence or slow convergence is indicated we will again bring to bear the Euler transformation with the hope of rendering the particular sum of interest (more rapidly) convergent.

4. Approximate solutions for the $g_{n}(\eta)$ from the solution representations of Eqs. (17) and (18). This section will be concerned with the approximate evaluation of the functions $g_{n}(\eta)$ by utilizing the exact quadrature form of solution presented in Eqs. (17) and (18). For a given $n$ attention is eventually focused here on the problem of obtaining a solution for the leading term $g_{n}(0)$ in the expansion of such a function. Once this has been obtained, all other terms in the expansion follow from readily accessible recurrence relations. In such a manner, somewhat similar to that sketched in the previous section, solutions for the $g_{n}(\eta)$ (and, of course, for the $b_{n}(\eta)$ ) can be constructed recursively.

We introduce the expansions of the $g_{n}$ as

TABLE 1

Solutions for the $b_{n}(0)=g_{n}(0)$.

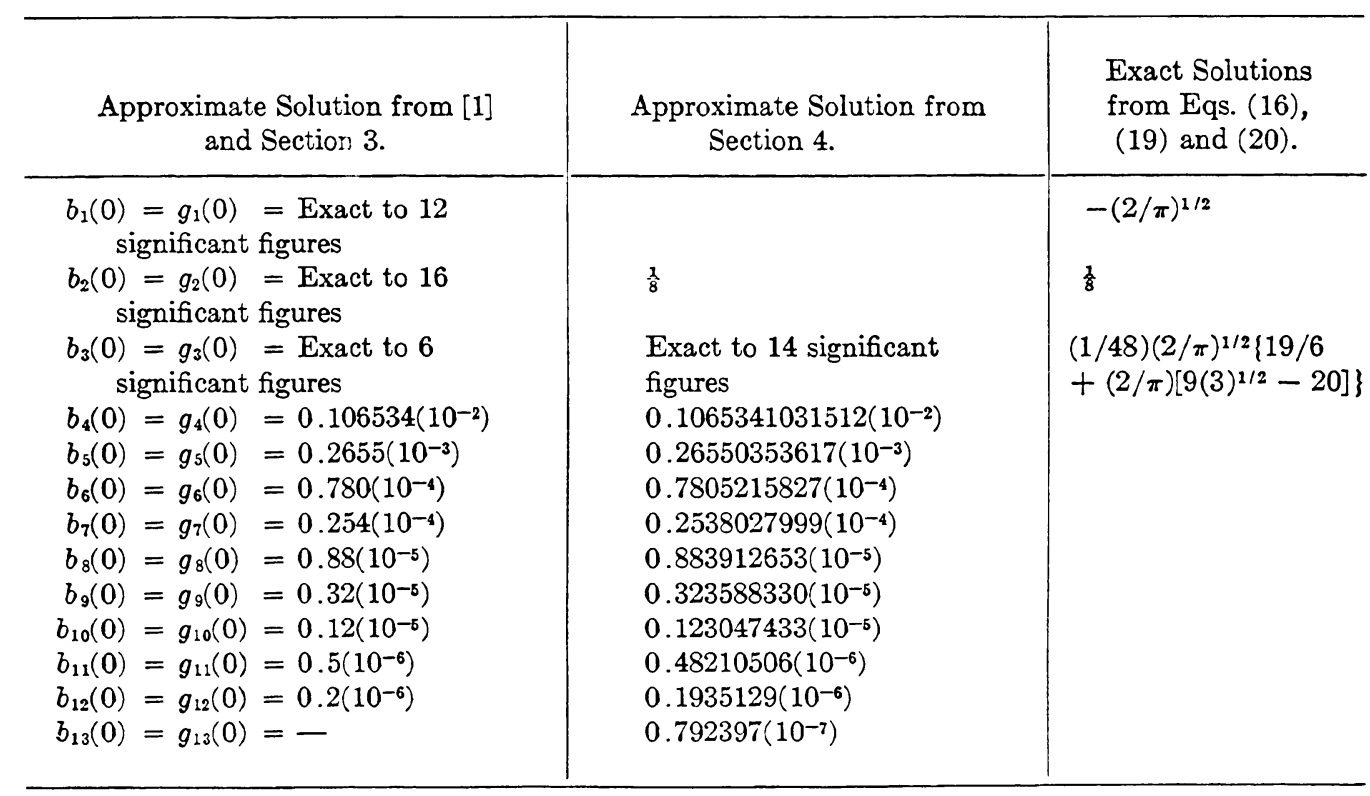




$$
g_{n}(\eta)=\sum_{m=0}^{\infty} A_{m, n}^{\prime} \eta^{m}
$$

For now we concentrate on a particular $g_{n}$ thereby assuming that all $g_{p}, p<n$ (i.e. all $\left.A_{m, p}^{\prime}, p<n\right)$ have been previously obtained. Taking the exact solution for $g_{1}(0)$ as per Eq. (16), we will be considering $n>1$. Note that the condition of Eq. (13) requires

$$
A_{1, n}^{\prime}=0, \quad n>1 \text {. }
$$

We proceed with our solution by considering an expansion of the entire function, $h_{n}(\eta)$, about $\eta=0$. In view of the definition of Eq. (15) and for later convenience, we take this expansion in the following form:

$$
h_{n}(\eta)=-\exp \left[-\eta^{2} / 4\right] \sum_{p=0}^{\infty}\left\{2^{p / 2+1} \Gamma[(p+n+3) / 2] K_{p, n} \eta^{p} / p !\right\} .
$$

From the form of the (known) functions $g_{p}, p<n$ as per Eq. (27) it is possible, from the definition of Eq. (15), to give explicit relationships for the $K_{p, n}$ as functions of the $A_{m, p}^{\prime}, p<n$. Thus

$$
\begin{aligned}
& K_{0, n}=\{2 \Gamma[(n+3) / 2]\}^{-1} \sum_{m=1}^{n-1} A_{0, m}^{\prime}\left(2 A_{2, n-m}^{\prime}-A_{0, n-m}^{\prime} / 2\right) ; \\
& K_{1, n}=\left\{2^{3 / 2} \Gamma[(n+4) / 2]\right\}^{-1}\left(-2 A_{0, n-1}^{\prime}+2 A_{2, n-1}^{\prime}+6 \sum_{m-1}^{n-1} A_{0, m}^{\prime} A_{3, n-m}^{\prime}\right), \\
& K_{p, n}=\left\{2^{p / 2+1} \Gamma[(p+n+3) / 2]\right\}^{-1}\left[p(p+1) A_{p+1, n-1}^{\prime}-A_{p-1, n-1}^{\prime} / 2\right. \\
&+ \sum_{m=1}^{n-1} A_{0, m}^{\prime}\left[(p+1)(p+2) A_{p+2, n-m}-(p+1 / 2) A_{p, n-m}^{\prime}\right] \\
&+\sum_{m=1}^{n-1} \sum_{a=0}^{p-2}\left\{A_{p-q, m}^{\prime}\left[(q+1)(q+2) A_{a+2, n-m}^{\prime}-A_{a, n-m}^{\prime} / 2\right]\right. \\
&\left.\left.-(q+1) A_{p-q-1, m}^{\prime} A_{a+1, n-m}^{\prime}+A_{p-a-2, m}^{\prime} A_{a, n-m}^{\prime} / 4\right\}\right], \quad p>1,
\end{aligned}
$$

where we have used the result of Eq. (28). According to the solution forms of Eqs. (17) and (18) we obtain the following exact solution for the unknown, $g_{n}(0)$, of interest:

$$
g_{n}(0)=-2^{n / 2} \pi^{-1 / 2} \Gamma[(n+1) / 2] \int_{0}^{\infty} D_{-n-1}(\mu) h_{n}(\mu) d \mu .
$$

We now insert the $h_{n}(\eta)$ expansion as per Eq. (29) into the integrands of (31). In view of the fact that this expansion is not valid throughout the entire range of integration (i.e. at $\eta \rightarrow \infty$ ) the equality sign is no longer necessarily valid. Formally exchanging the order of summation and integration we finally obtain

$$
\begin{aligned}
& g_{n}(0) \sim 2^{n / 2} \pi^{-1 / 2} \Gamma[(n+1) / 2] \sum_{p=0}^{\infty} 2^{p / 2+1}\{\Gamma[(p+n+3) / 2] / p !\} K_{p, n} \\
& \cdot \int_{0}^{\infty} \mu^{p} \exp \left(-\mu^{2} / 4\right) D_{-n-1}(\mu) d \mu .
\end{aligned}
$$


We have again utilized the $\sim$ sign to indicate that the right-hand side of (32) is only a formally obtained representation for the $g_{n}(0)$. Now the integrals of (31) for the case $p \geq 0$ of interest have been exactly given [6] as

$$
\int_{0}^{\infty} \mu^{p} \exp \left(-\mu^{2} / 4\right) D_{-n-1}(\mu) d \mu=\pi^{1 / 2} 2^{-\not / 2-n / 2-1} p ! / \Gamma[(p+n+3) / 2] .
$$

Using this in (32) we obtain

$$
g_{n}(0) \sim \Gamma[(n+1) / 2] \sum_{p=0}^{\infty} K_{p, n} .
$$

The problem has now become one of obtaining a meaningful equation for the $g_{n}(0)$ from the representation of Eq. (33). The situation is similar to the one encountered and briefly discussed in the previous section. The relationship of Eq. (25) in that section corresponds, in this regard, to Eq. (33). However, as pointed out earlier, the terms of the infinite sum on the left side of Eq. (25) contain the unknown, $b_{n}(0)$, of interest whereas the terms $K_{p, n}$ on the left side of Eq. (33) are independent of the present unknown, $g_{n}(0)$, and can be determined from Eq. (30). In the present situation the Euler transformation of Eqs. (26) will again be utilized to force convergence or more rapid convergence of the sums of Eq. (33). Thus Eq. (33) will directly result in a solution for $g_{n}(0)$. Once $g_{n}(0)=A_{0, n}^{\prime}$ has been obtained it is a simple matter to obtain all of the other $A_{p, n}^{\prime}$. These follow from a recursion relationship deduced from Eq. (12) with the use of the expansions of Eqs. (27) and (29) and the result of Eq. (28). Thus

$$
\begin{aligned}
A_{0, n}^{\prime}= & g_{n}(0), \quad A_{1, n}^{\prime}=0, \quad A_{2, n}^{\prime}=(2 n+1) A_{0, n}^{\prime} / 4-\Gamma[(n+3) / 2] K_{0, n}, \\
A_{3, n}^{\prime}= & -2^{1 / 2} \Gamma[(n+4) / 2] K_{1, n} / 3, \\
A_{p, n}^{\prime}= & {[p(p-1)]^{-1}\left\{(2 n+1) A_{p-2, n}^{\prime} / 2+A_{p-4, n}^{\prime} / 4\right.} \\
& \left.-\sum_{q=0}^{P / 2-1}(-1)^{a} \Gamma[(p+n+1) / 2-q] K_{p-2-2 q, n} 2^{p / 2-3 q} /[q !(p-2-2 q) !]\right\}, p>3,
\end{aligned}
$$

where

$$
\begin{aligned}
& P / 2=p / 2 \quad \text { if } \quad p \quad \text { is even } \\
& =(p-1) / 2 \text { if } p \text { is odd, and } n>1 .
\end{aligned}
$$

With all of the above in mind we now proceed actually to obtain the $g_{n}(0)=A_{0, n}^{\prime}$ and, in fact, the $A_{p, n}^{\prime}, n>1$ in general. Using the exact result for $g_{1}(\eta)$ as given in Eq. (16) we proceed to evaluate the subsequent $g_{n}$ one at a time starting with $g_{2}$.

$n=2$. In order to evaluate $g_{2}(0)$ from Eq. (33) we require the $K_{p, 2}$ as given in Eq. (30). These depend on the $A_{p .1}^{\prime}$, which, according to Eq. (16) and the expansion of Eq. (27), are exactly given by [8]

$$
\begin{aligned}
A_{2 k, 1}^{\prime} & =(2 / \pi)^{1 / 2} \sum_{a=0}^{k}(-1)^{\circ}\left[(2 q-1) 2^{2 k-a} q !(k-q) !\right]^{-1}, \\
A_{2 k+1,1}^{\prime} & =2^{-2 k} / k ! .
\end{aligned}
$$

Using these last in Eqs. (30) all $K_{p, 2}$ can be evaluated. For the present calculation, however, it is more direct to evaluate the $K_{p, 2}$ by using an exact solution for $h_{2}(\eta)$. 
This can be obtained from the definition of Eq. (15) with the use of the exact solution for $g_{1}(\eta)$ as given by Eq. (16). Thus we obtain

$$
\begin{aligned}
h_{2}(\eta) & =-(2 / \pi) D_{0}^{2}(\eta) D_{-2}(\eta) \\
& =-\exp \left[-\eta^{2} / 4\right]\left\{(2 / \pi) \exp \left[-\eta^{2} / 2\right]-(2 / \pi)^{1 / 2} \eta+(2 / \pi)^{1 / 2} \eta \operatorname{erf}\left[\eta / 2^{1 / 2}\right]\right\},
\end{aligned}
$$

where the error function, erf $(x)$, is defined and expanded as [8]

$$
\begin{aligned}
\operatorname{erf} & (x)=\left(2 / \pi^{1 / 2}\right) \int_{0}^{x} \exp \left[-t^{2}\right] d t \\
= & \left(2 / \pi^{1 / 2}\right) \sum_{k=1}^{\infty}(-1)^{k+1} x^{2 k-1}[(2 k-1)(k-1) !]^{-1} .
\end{aligned}
$$

In view of the above we find that $h_{2}$ can be given by

$$
h_{2}(\eta)=-\exp \left[-\eta^{2} / 4\right]\left\{-(2 / \pi)^{1 / 2} \eta-(2 / \pi) \sum_{k=0}^{\infty}(-1)^{k} \eta^{2 k}\left[k ! 2^{k}(2 k-1)\right]^{-1}\right\} .
$$

Comparing this with the $h_{2}$ expansion of Eq. (29), and equating like coefficients of $-\eta^{p} \exp \left[-\eta^{2} / 4\right]$, we immediately obtain

$$
\begin{aligned}
K_{2 k, 2} & =-4(-1)^{k} \pi^{-3 / 2}[(2 k-1)(2 k+1)(2 k+3)]^{-1}, \quad k \geq 0, \\
K_{1,2} & =-\pi^{-1 / 2} / 4, \text { all other } K_{2 k+1,2}=0 .
\end{aligned}
$$

Using these results in Eq. (33) the following representation for $g_{2}(0)$ results:

$$
\begin{aligned}
g_{2}(0) & \sim\left(\pi^{1 / 2} / 2\right) \sum_{p=0}^{\infty} K_{p, 2} \\
& =-1 / 8-(2 / \pi) \sum_{k=0}^{\infty}(-1)^{k}[(2 k-1)(2 k+1)(2 k+3)]^{-1}=1 / 8,
\end{aligned}
$$

where the result $[8] \sum_{k=0}^{\infty}(-1)^{k} /(2 k+1)=\pi / 4$ has been used.

As it happens, the above result indicates that the infinite sum $\sum K_{p, 2}$ is not only convergent, but it is also summable in closed form. Moreover, as is evident from Eq. (19), the representation for $g_{2}(0)$ as given by Eq. (33) yields the exact solution for this quantity. As will be seen, the convergence of the infinite sums $\sum K_{p, n}$ of Eq. (33) is not generally in evidence. In our computation of subsequent $g_{n}(0)$ we will therefore incorporate the Euler transformation in an attempt to force convergence or more rapid convergence of the particular $\sum K_{p, n}$ under consideration. With regard to the present calculation we point out that the use of the single Euler transformation on the series $\sum K_{\mathfrak{p}, 2}$ appears to render this series more rapidly convergent. Thus, assuming that the closed-form sum of this series was not at our disposal, we would have obtained our value for $g_{2}(0)$ from

$$
g_{2}(0) \sim\left(\pi^{1 / 2} / 2\right) \sum_{p=0}^{Q} K_{p .2} .
$$

Without any further values of the $K_{p, 2}$, however, this approximation can be improved by taking

$$
g_{2}(0) \sim\left(\pi^{1 / 2} / 2\right) \sum_{p=0}^{q} \varepsilon^{m}\left(K_{p, 2}\right)
$$


for some optimum value of $m$. This is evident from a comparison between the approximate results obtained from Eqs. (37) and (38) for various values of $q$ and $m$ as given in Table 2. This table indicates that for the present calculation the most judicious choice of $m$ appears to be $m=1$. Using double precision accuracy on the G.E. 635 computer it was possible to establish an approximate result for $g_{2}(0)$ accurate to 17 significant figures by using $m=1$ and $q=78$ in Eq. (38). Using the same $q=78$ and Eq. (37) one obtains a result for $g_{2}(0)$ accurate to only 5 significant figures.

$n=3$. Using the exact result $g_{2}(0)=\frac{1}{8}$, and the solutions for $K_{p, 2}$ as given in Eq. (46), the coefficients $A_{p, 2}^{\prime}$ can be computed from Eq. (34). With these at our disposal along with the $A_{p, 1}$ as per Eq. (35), the $K_{p, 3}$ can be computed from Eq. (30). As with $g_{2}(0)$, it is expected that the solution for $g_{3}(0)$ can be ascertained from the representation of Eq. (33). Thus, for large enough $q$, we expect that either the representation

$$
g_{3}(0) \sim \sum_{p=0}^{Q} K_{p, 3}
$$

will yield an accurate solution for $g_{3}(0)$, or else, for some $m$, the representation

TABLE 2

Evaluation of $g_{2}(0)$ from Eqs. (37) and (38).

\begin{tabular}{rrrrr}
\hline & $g_{2}(0) \sim$ & $g_{2}(0) \sim$ & $g_{2}(0) \sim$ \\
\hline & & & & \\
\hline & $\Gamma\left(\frac{3}{2}\right) \sum_{p=0}^{q} K_{p, 2}=$ & $\Gamma\left(\frac{3}{2}\right) \sum_{p=0}^{q} \mathcal{E}^{(1)}\left(K_{p, 2}\right)=$ & $\Gamma\left(\frac{3}{2}\right) \sum_{p=0}^{q} \varepsilon^{(2)}\left(K_{p, 2}\right)=$ & $\Gamma\left(\frac{3}{2}\right) \sum_{p=0}^{q} \varepsilon^{(3)}\left(K_{p, 2}\right)=$ \\
0 & $.212206_{6}$ & $.106103_{3}$ & $.053051_{6}$ & $.026525_{8}$ \\
1 & $.087206_{6}$ & $.127904_{9}$ & $.085027_{9}$ & $.047782_{8}$ \\
2 & $.129647_{9}$ & $.128485_{9}$ & $.103813_{8}$ & $.064756_{6}$ \\
3 & $.129647_{9}$ & $.126269_{1}$ & $.114503_{5}$ & $.078258_{3}$ \\
4 & $.123584_{9}$ & $.125043_{8}$ & $.120338_{0}$ & $.088954_{1}$ \\
5 & $.123584_{9}$ & $.124751_{7}$ & $.123343_{2}$ & $.097389_{5}$ \\
6 & $.125605_{9}$ & $.124829_{1}$ & $.124759_{1}$ & $.104010_{2}$ \\
7 & $.125605_{9}$ & $.124947_{9}$ & $.125325_{4}$ & $.109179_{5}$ \\
8 & $.124687_{2}$ & $.125010_{0}$ & $.125469_{3}$ & $.113192_{2}$ \\
9 & $.124687_{2}$ & $.125022_{4}$ & $.125427_{5}$ & $.116287_{4}$ \\
10 & $.125181_{9}$ & $.125013_{8}$ & $.125323_{4}$ & $.118657_{8}$ \\
11 & $.125181_{9}$ & $.125003_{6}$ & $.125215_{6}$ & $.120458_{8}$ \\
12 & $.124885_{1}$ & $.124998_{4}$ & $.125127_{6}$ & $.121814_{7}$ \\
13 & $.124885_{1}$ & $.124997_{5}$ & $.125064_{7}$ & $.122824_{7}$ \\
14 & $.125077_{1}$ & $.124998_{5}$ & $.125024_{6}$ & $.123567_{8}$ \\
15 & $.125077_{1}$ & $.124999_{7}$ & $.125001_{8}$ & $.124106_{4}$ \\
16 & $.124945_{7}$ & $.125000_{2}$ & $.124990_{9}$ & $.124489_{6}$ \\
17 & $.124945_{7}$ & $.125000_{3}$ & $.124987_{4}$ & $.124756_{1}$ \\
18 & $.125039_{6}$ & $.125000_{2}$ & $.124987_{9}$ & $.124935_{5}$ \\
19 & $.125039_{6}$ & $.125000_{0}$ & $.124990_{2}$ & $.125051_{2}$ \\
20 & $.124970_{2}$ & $.125000_{0}$ & $.124993_{0}$ & $.125120_{8}$ \\
21 & $.124970_{2}$ & $.125000_{0}$ & $.124995_{5}$ & $.125157_{8}$ \\
22 & $.125023_{0}$ & $.125000_{0}$ & $.124997_{5}$ & $.125172_{3}$ \\
23 & $.125023_{0}$ & $.125000_{0}$ & $.124998_{9}$ & $.125171_{8}$ \\
24 & $.124981_{9}$ & $.125000_{0}$ & $.124999_{8}$ & $.125162_{0}$ \\
25 & $.124981_{9}$ & $.125000_{0}$ & $.125000_{3}$ & $.125146_{7}$ \\
\hline & & & &
\end{tabular}




$$
g_{3}(0) \sim \sum_{p=0}^{e} \varepsilon^{(m)}\left(K_{p, 3}\right)
$$

will optimize the accuracy of the unknown $g_{3}(0)$ under consideration. For several different $q$, Table 3 shows the values of $g_{2}(0)$ resulting from the representation of Eq. (39) and from the representation of Eq. (40) for different $m$. From this table it appears that of the representations considered, the optimum accuracy for $g_{3}(0)$ might be expected from the representation of Eq. (40) using $m=1$ or 2 . From the results of the first column of this table it is clear that no useful information regarding the value of our unknown, $g_{3}(0)$, has been obtained directly from the representation of Eq. (39). By using double precision accuracy on the G.E. 635 computer it was possible, however, to establish an approximate result for $g_{3}(0)$ accurate to 14 significant figures by using $m=2$ and $q=112$ in Eq. (40). It is of interest to note that with $q=112$, Eq. (40) yields 11-place accuracy with $m=1$ and 9 -place accuracy with $m=3$. It is worthwhile to note further that while $q=112$ is required for 14-place accuracy in $g_{3}(0), q=78$ was sufficient for 17-place accuracy in the computation for $g_{2}(0)$. For a given $q$ there is a persistent loss

TABLE 3

Evaluation of $g_{3}(0)$ from Eqs. (39) and (40).

\begin{tabular}{|c|c|c|c|c|}
\hline & $g_{3}(0) \sim$ & $g_{3}(0) \sim$ & $g_{3}(0) \sim$ & $g_{3}(0) \sim$ \\
\hline$q=$ & $\Gamma(2) \sum_{p=0}^{q} K_{p, 3}$ & $\Gamma(2) \sum_{p=0}^{q} \mathcal{E}^{(1)}\left(K_{p, 3}\right)$ & $\Gamma(2) \sum_{p=0}^{q} \varepsilon^{(2)}\left(K_{p, 3}\right)$ & $\Gamma(2) \sum_{p=0}^{q} \mathcal{E}^{(3)}\left(K_{p, 3}\right)$ \\
\hline 0 & .052185 & $.026092_{7}$ & $.013046_{4}$ & $.006523_{2}$ \\
\hline 1 & $-.056671_{3}$ & $.011925_{0}$ & $.016027_{3}$ & $.010530_{1}$ \\
\hline 2 & $.048378_{8}$ & $.004365_{2}$ & $.014802_{3}$ & $.012753_{1}$ \\
\hline 3 & $.001725_{8}$ & $.003997_{2}$ & $.012336_{2}$ & $.013743_{6}$ \\
\hline 4 & $-.012625_{8}$ & $.005437_{7}$ & $.009962_{2}$ & $.013911_{7}$ \\
\hline 5 & $.017357_{1}$ & $.006220_{4}$ & $.008149_{1}$ & $.013557_{8}$ \\
\hline 6 & $.014182_{5}$ & .006034 g & $.006951_{1}$ & $.012898_{1}$ \\
\hline 7 & $-.008174_{8}$ & $.005906_{z}$ & $.006256_{2}$ & $.012084_{1}$ \\
\hline 8 & $.009109_{6}$ & $.005897_{6}$ & $.005912_{4}$ & $.011219_{5}$ \\
\hline 9 & $.011222_{8}$ & $.005936_{2}$ & $.005784_{\mathrm{s}}$ & $.010372_{0}$ \\
\hline 10 & $.006460_{8}$ & $.005963_{8}$ & $.005771_{8}$ & $.009583_{2}$ \\
\hline 11 & $-.002502_{2}$ & $.005967_{4}$ & $.005808_{3}$ & $.008876_{1}$ \\
\hline 12 & $.010253_{2}$ & $.005959_{0}$ & $.005858_{0}$ & $.008260_{7}$ \\
\hline 13 & $.010501_{1}$ & .005951 g & $.005901_{2}$ & $.007738_{1}$ \\
\hline 14 & $.003781_{1}$ & $.005950_{4}$ & $.005932_{1}$ & $.007304_{0}$ \\
\hline 15 & $-.001172_{7}$ & $.005952_{5}$ & $.005950_{7}$ & $.006950_{6}$ \\
\hline 16 & .011430 & $.005954_{6}$ & $.005959_{8}$ & $.006668_{6}$ \\
\hline 17 & $.011336_{6}$ & $.005955_{2}$ & $.005962_{7}$ & $.006448_{1}$ \\
\hline 18 & $.001160_{5}$ & $.005954_{7}$ & $.005962_{1}$ & $.006279_{2}$ \\
\hline 19 & $-.002000_{3}$ & $.005954_{0}$ & $.005960_{0}$ & $.006153_{0}$ \\
\hline 20 & $.014035_{8}$ & $.005953_{7}$ & $.005957_{8}$ & $.006061_{0}$ \\
\hline 21 & $.013873_{1}$ & $.005953_{8}$ & $.005955_{9}$ & $.005996_{4}$ \\
\hline 22 & $-.003232_{0}$ & $.005954_{1}$ & $.005954_{7}$ & $.005952_{8}$ \\
\hline 23 & $-.005428 s$ & $.005954_{2}$ & .005953 g & $.005925_{2}$ \\
\hline 24 & $.019863_{1}$ & $.005954_{2}$ & $.005953_{6}$ & .005909 \\
\hline 25 & $.019698_{2}$ & $.005954_{1}$ & $.005953_{5}$ & $.005902_{1}$ \\
\hline
\end{tabular}


of significant figures in the computation for a particular $g_{n}(0)$ as $n$ increases. Moreover, in such computations for a given $g_{n}(0)$ full use is made of the available accuracy of all preceding $g_{n}(0)$ 's. Thus the higher the accuracy of early $g_{n}(0)$ 's the more $g_{n}(0)$ 's can be computed. It is for this reason that it is useful to obtain as much accuracy as an available computer facility will allow in the computation of early $g_{n}(0)$ 's.

With regard to the above approximate computations for the (known) values of $g_{2}(0)$ and $g_{3}(0)$, the following indicated rules are noted in summary:

1. When the infinite sums of Eq. (33), representative of the values of $g_{n}(0)$, are truncated to finite sums, there is a significant improvement in the accuracy of the representation when the single or multiple Euler transformation of these sums are utilized.

2. For a given $n$ (within the scope of the calculations which were performed), a representative $g_{n}(0)$ partial sum, $\sum_{0}$, obtained from Eq. (33), and the sums, $\sum_{1}$, $\sum_{2}, \cdots$, obtained by the various multiple Euler transformations of such a sum, all yield approximate values for $g_{n}(0)$ accurate to within the order of magnitude of the "final terms" of the particular sum, $\sum_{r}$, being considered.

For a given $n$, the optimum number of transformations, $m$, can be concluded by invoking rule 2. Thus, $m=m(n)$. In particular, the above computations have yielded the results $m(2)=1$ and $m(3)=2$.

$n>3$. We now extend rules 1 and 2 above, and assume that they are valid for the approximate computation of all the $g_{n}(0)$. With such an assumption further results have been obtained for all $g_{n}(0)$ up to $g_{13}(0)$, and these are presented on Table 1 along with the approximate results for $g_{n}(0), n=1,2$ and 3 , as obtained earlier in this section. It is of interest to note that these new results were obtained from the general representation

$$
g_{n}(0)=\Gamma[(n+1) / 2] \sum_{p=0}^{o(n)} \mathcal{E}^{[m(n)]}\left(K_{p, n}\right)
$$

where the number of transformations, $m$, for optimum accuracy was found to be

$$
\begin{aligned}
m(2) & =1, \quad m(3)=m(4)=m(5)=m(6)=m(7)=m(8)=m(9)=2, \\
m(10) & =m(11)=m(12)=m(13)=3 .
\end{aligned}
$$

5. Evaluation of $\phi(\eta, \zeta)$. From the solution form for $\phi$ as given in Eq. (6) along with the definition of Eq. (11), the exact results of Eqs. (7), (16), (19), and (20), and the approximate results for the $A_{m, n}^{\prime}$ of the previous section, we consider an evaluation of our unknown function from the representation

$$
\begin{aligned}
\phi & =1+\left[\sum_{n=1}^{\infty} g_{n}(\eta) \zeta^{n}\right] \exp \left(-\eta^{2} / 4\right) \\
& =1+\left[g_{1}(\eta) \zeta+g_{2}(\eta) \zeta^{2}+g_{3}(\eta) \zeta^{3}+\sum_{r=4}^{\infty}\left(\sum_{s=0}^{\infty} A_{r, 8}^{\prime} \eta^{\circ}\right) \zeta^{r}\right] \exp \left(-\eta^{2} / 4\right) .
\end{aligned}
$$

In the actual computation, the internal sums $\sum_{:-0}^{\infty} A_{r, 8}^{\prime} \eta^{s}=g_{r}(\eta), r>3$ are truncated, $s$ going from 0 to 99 . The index $r$ in the second summation is taken from 4 to 13. The error of the results in these computations, first for the $g_{r}(\eta)$, and, finally, for $\phi(\eta, \zeta)$ is estimated from the order of magnitude of the latter terms of the partial sums con- 
sidered. A plot of $\phi(\eta, \zeta)$ obtained through use of the above calculation procedure is given in the solid lined curves of Fig. 1. This graphical presentation of $\phi$ (solid curves) is plotted from calculated results which are estimated to be less than $1 \%$ in error.

Since the temperature history of the halfspace as a function of time and depth is probably the most useful physical result of our solution, this has been obtained from our above mentioned calculations for $\phi$ and from our definition (1) of the dimensionless temperature $V$. The result is presented in the solid lined curves of Fig. 2 where $V$ is plotted as a function of $\lambda H|\lambda H| t$ with $|\lambda H| x$ as a parameter. Here the dimensionless quantities $x, t$ and $H$ may be defined, as in [1], by

$$
x=x^{*}\left(T_{0}^{*} C^{* 3}\right)^{1 / 2} \rho^{*} / k_{0}^{*} ; t=t^{*} T_{0}^{*} C^{*^{2}} \rho^{*} / k_{0}^{*} ; H=H^{*} /\left[\left(T_{0}^{*} C^{*}\right)^{3 / 2} \rho^{*}\right] .
$$

The solid lined curves of Figs. 1 and 2 are also representative of plots of solutions for $\phi$ and $V$ which have been obtained from an approximation for $\phi$ as per Eq. (42) with the infinite sum over $n$ truncated after only 3 terms (i.e. using only the exact solutions $g_{1}, g_{2}$, and $g_{3}$ ). Where such solution representations differ from our earlier evaluations they are presented in dashed lines. As seen, such a readily usable approximate closed-form solution gives good accuracy in $\phi$ or $V$ for a significant range of the $\zeta$ or $\lambda H|\lambda H| t$ variables respectively.

6. Results and conclusions. Since the problem of the present work and that of [1] are the same, the plots of our solution function are identical in the pertinent range of

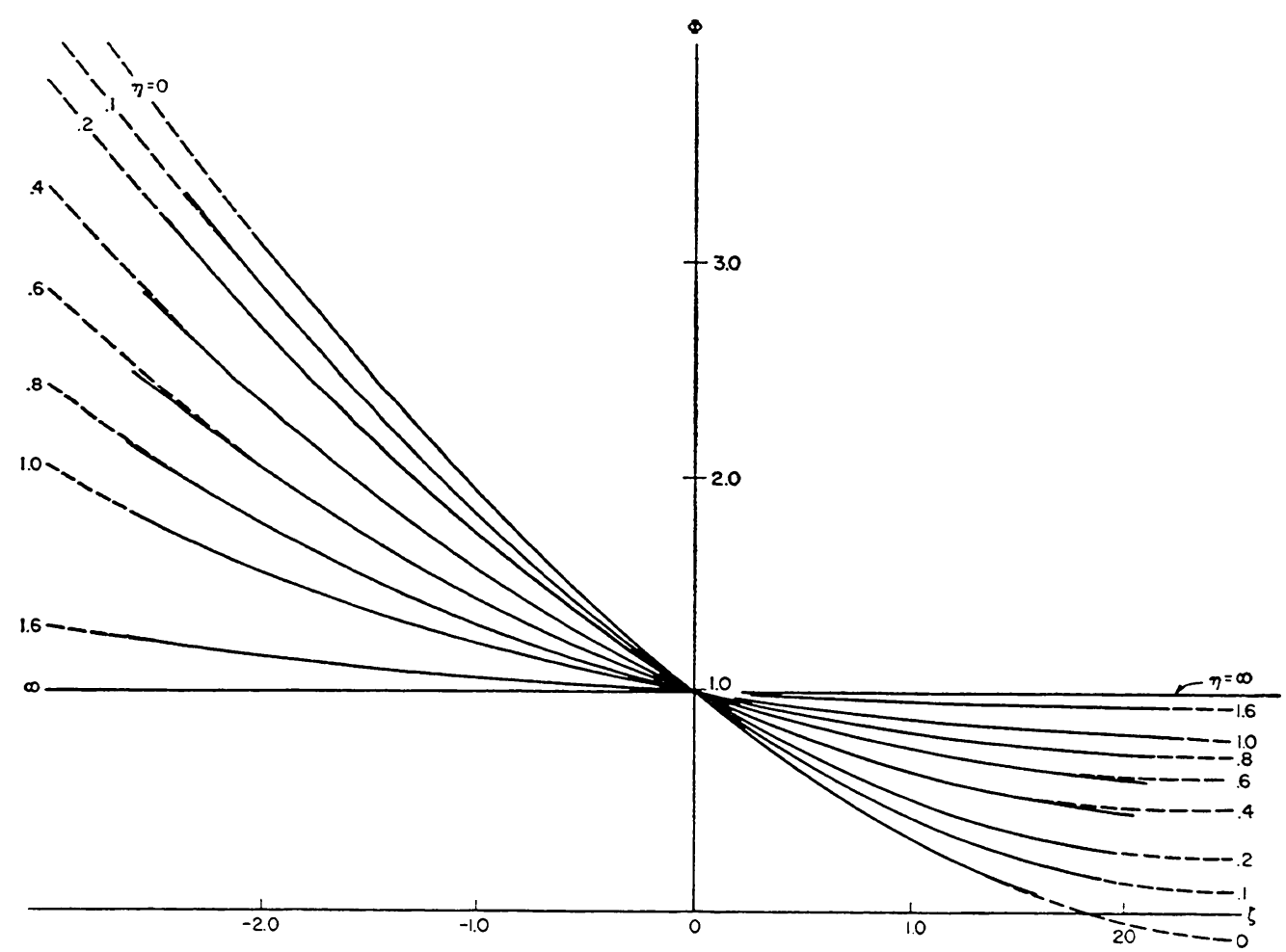

Fig. 1. The dimensionless conductivity, $\phi$, as a function of $\zeta$ with $\eta$ as a parameter. 


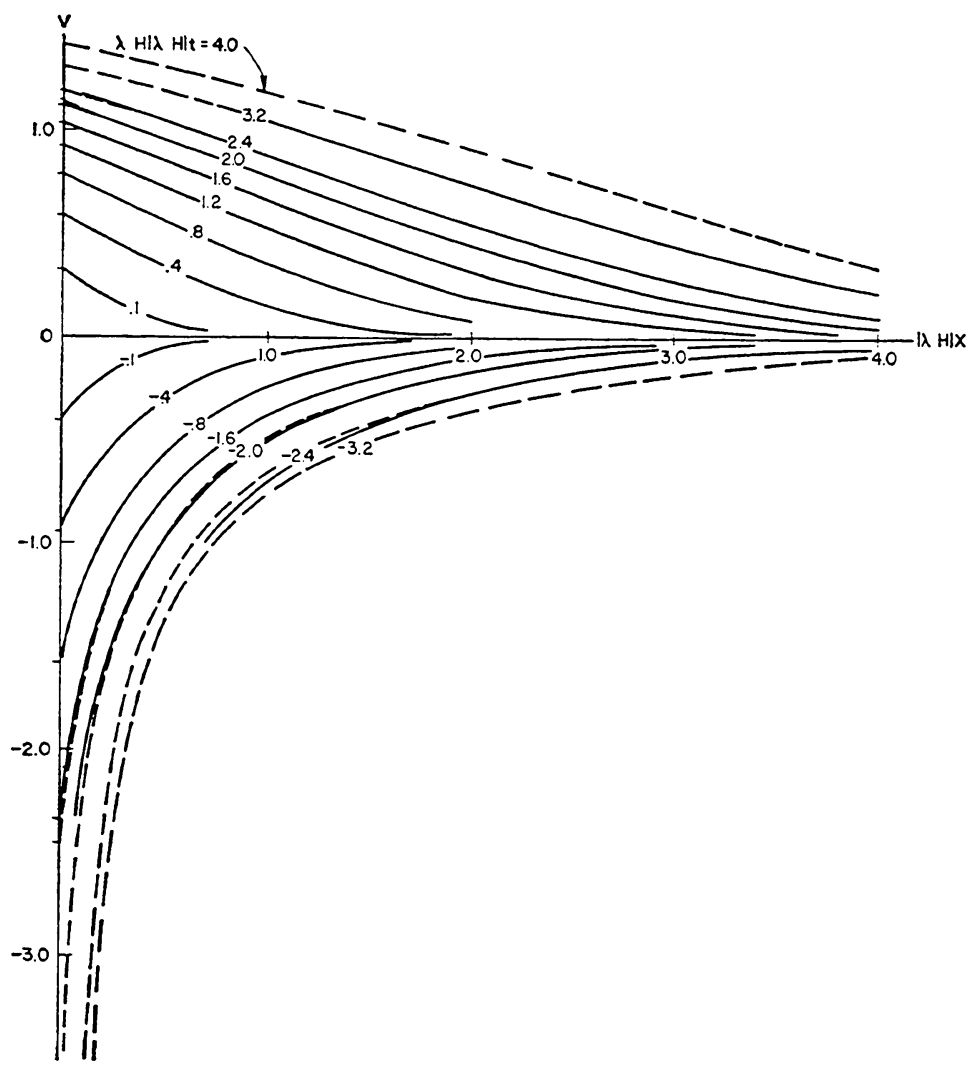

Fig. 2. The dimensionless temperature, $V$, as a function of $|\lambda H| x$ with $\lambda H|\lambda H| t$ as a parameter.

independent variables to those plots obtained in [1]. The improved solution technique developed here along with the more extensive calculations have, however, resulted in evaluations for $\phi$ and $V$ which are generally more accurate than those of [1] even though these improvements do not show up in the relatively rough solution plots which are presented.

For an interpretation of our solution vis-à-vis the physical phenomenon of the heated (or cooled) halfspace the reader is referred to the discussion at the end of [1] which is entirely pertinent to the present work.

With regard to the solution technique of this study the following summary and conclusions are finally noted:

1. The new exact solutions for $g_{2}(\eta)$ and $g_{3}(\eta)$ (or $b_{2}(\eta)$ and $b_{3}(\eta)$ ) obtained in Sec. 2 along with the proof of analyticity of the general functions $g_{n}(\eta)$ or $b_{n}(\eta)$ gives us increased confidence in the approximate solution techniques and results of both reference [1] and the present work.

2. The approximate solution representation obtained from Eqs. (6) or (32), where we only consider terms up to and including $O\left(\zeta^{3}\right)$, is useful over a significant range of the independent variables. 


\section{REFERENCES}

[1] L. Y. Cooper, Constant heating of a variable conductivity halfspace, Quart. Appl. Math. 27, 173-184 (1969)

[2] D. Meksyn, New methods in laminar boundary layer theory, Pergamon Press, London, 1961

[3] H. Görtler, A new series for the calculation of steady laminar boundary layer flows, J. Math. Mech. 6, 1-66 (1957)

[4] H. Görtler, On the calculation of steady laminar boundary layer flows with continuous suction, J. Math. Mech. 6, 323-340 (1957)

[5] E. Kamke, Differentialgleichungen lösungsmethoden und lösungen, Chelsea, New York, 1959.

[6] A. Erdélyi et al., Higher transcendental functions. Vol. II, McGraw-Hill, New York, 1953

[7] J. B. Rosser, Transformations to speed the convergence of series, J. Res. Nat. Bureau Standards 46, $56-64$ (1951)

[8] I. S. GradšteIn and I. M. Ryžik, Tables of integrals, series and products, Academic Press, New York, 1951 